\title{
Emotion Regulation and the Quality of Social Interaction: Does the Ability to Evaluate Emotional Situations and Identify Effective Responses Matter?
}

\author{
Paulo N. Lopes, ${ }^{1}$ John B. Nezlek, ${ }^{2}$ Natalio Extremera, ${ }^{3}$ \\ Janine Hertel, ${ }^{4}$ Pablo Fernóndez-Berrocal, ${ }^{3}$ Astrid Schütz, ${ }^{4}$ \\ and Peter Salovey ${ }^{5}$ \\ ${ }^{1}$ Catholic University of Portugal \\ ${ }^{2}$ College of William and Mary, USA \\ ${ }^{3}$ University of Málago, Spain \\ ${ }^{4}$ Chemnitz University of Technology, Germany \\ ${ }^{5}$ Yale University, USA
}

\begin{abstract}
We examined self and friends' ratings of social relationship quality and everyday social interactions in 3 studies involving 544 college students in Germany, Spain, and the United States. Scores on a situational judgment test measuring strategic emotion regulation ability (SERA) were negatively related to conflict with others. SERA was more consistently and strongly related to conflict with others than to the positive dimension of relationship quality (support, companionship, and nurturance). The relationship between SERA and conflict was generally not mediated by trait positive or negative affect, and it remained significant or marginally significant controlling for the Big Five personality traits. These findings highlight the importance of the ability to evaluate emotional situations and identify effective responses to these in interpersonal emotion regulation. Furthermore, they suggest that situational judgment and flexible response selection may help people to manage conflicts more than to bond with others.
\end{abstract}

This article examines how the ability to evaluate emotional situations and identify effective response strategies to manage these

We thank Rosario Cabello for allowing us to use data that she collected together with Pablo Fernández-Berrocal for our meta-analytic synthesis of findings.

Correspondence concerning this article should be addressed to Paulo N. Lopes, Catholic University of Portugal, Faculty of Economics and Management, Palma de Cima, Lisbon 1649-023, Portugal. Email: paulo.lopes@fcee.ucp.pt.

Journal of Personality 79:2, April 2011

(C) 2011 The Authors

Journal of Personality (C) 2011, Wiley Periodicals, Inc.

DOI: $10.1111 / \mathrm{j} .1467-6494.2010 .00689 . x$ 
situations is related to the quality of interpersonal relationships and social interaction. We view this dimension of emotion regulation as an important aspect of people's capacity to manage emotions in themselves and in others, integrating emotion and cognition. In prior research based on Mayer and Salovey's (1997; Salovey \& Mayer, 1990) model of emotional intelligence, this ability has generally been labeled simply as "emotion regulation" or "managing emotions." To distinguish it from other conceptualizations and measures of emotional regulation, and because this ability entails using one's knowledge and intelligence to evaluate emotional situations and decide how to act or respond, we will refer to it as "strategic emotion regulation." We acknowledge that there are other valid ways of conceptualizing and defining emotional regulation (e.g., Gross, 1998b). Nonetheless, we think that this dimension of emotion regulation ability merits specific attention.

Functional perspectives on emotion suggest that affective responses help individuals to adapt to and navigate the social environment. Emotions can guide thinking and motivate action (e.g., Frijda, 1988; Isen, 1987), and they convey information about people's thoughts and intentions (Ekman, 2003). They facilitate communication and help to coordinate social encounters through emotional contagion, social appraisal, and interpersonal reinforcement (Keltner \& Haidt, 2001; Parkinson, Fischer, \& Manstead, 2005). Nevertheless, there are times when emotions need to be regulated. For example, when furious, people may say and do things they later regret, damaging relationships. Intense emotions can undermine rational decision making by interrupting complex information processing or triggering counterproductive automatic responses (Simon, 1967). Emotions can also bias interpretations of ambiguous situations and risk judgments, leading to poor decisions (Lerner \& Tiedens, 2006).

Converging evidence from several lines of research suggests that emotional balance and regulation are associated with the quality and stability of social relationships (e.g., Caspi, 2000; Eisenberg, Fabes, Guthrie, \& Reiser, 2000; Kagan, 1998). Children who display more intense negative emotions and have difficulties regulating them interact less competently with peers than better-regulated counterparts (e.g., Eisenberg \& Fabes, 1999; Eisenberg et al., 1993). People like to interact with individuals who experience and express positive affect (Harker \& Keltner, 2001; Lyubomirsky, King, \& Diener, 2005) and 
avoid those who experience and express negative emotions persistently (Furr \& Funder, 1998). Effective conflict management also requires emotion regulation (Mischel \& DeSmet, 2000). In conflict situations, emotional arousal from repeated provocations (Zillmann, 1993) can cause people to vent anger and reciprocate destructive behavior, which in turn increases the probability of escalation in conflicts (Arriaga \& Rusbult, 1998). Last but not least, individuals may be ostracized if they do not modulate their emotional responses in accordance with prevailing feeling and display rules (Eid \& Diener, 2001; Ekman, 2003).

The ability to regulate emotions in the self and in others is considered to be a crucial dimension of emotional intelligence. The ability model of emotional intelligence proposed by Mayer and Salovey (1997; Salovey \& Mayer, 1990) emphasizes the importance of integrating emotion and cognition to adapt to life circumstances, to make sound decisions, and to attain personal goals. In this model, emotional abilities involve the capacity to use reasoning and prior knowledge to process emotional information.

Gross (1998b, p. 275) defined emotion regulation as a dynamic process "by which individuals influence which emotions they have, when they have them, and how they experience and express these emotions." People differ in their ability to identify effective regulation strategies, access these strategies, and use them flexibly (e.g., Cole, Michel, \& Teti, 1994; Thompson, 1994), and individual differences in this ability are thought to influence adaptation in various life domains. There is evidence that antecedent-focused emotion regulation, which takes effect before an individual becomes fully aroused, is more effective than response-focused emotion regulation, which seeks to modulate emotional expression or behavior when an individual is already experiencing emotional arousal (Gross, 1998a, 1998b). Strategic emotion regulation, as defined above, encompasses two key dimensions of antecedent-focused regulation: the way people evaluate emotional situations (appraisal) and identify responses to influence these situations (situation modification).

Although the social information-processing model (revised by Crick \& Dodge, 1994) emphasizes cognition rather than emotion, the findings from this line of research are relevant for our purposes. In particular, this research suggests that social maladjustment often stems from biases in the interpretation of ambiguous social situations and deficits in the selection of appropriate responses (see review 
by Crick \& Dodge). For example, aggressive and socially rejected children and adolescents often tend to attribute hostile intent to others in ambiguous situations, believe that the best way to respond to provocation is to strike back, have a limited response repertoire, and fail to consider alternative response strategies. This body of theory and research thus supports our focus on situation evaluation and response identification because it, too, emphasizes individuals' appraisals of social situations and the planning of responses.

\section{Research Using Ability Measures of Emotion Regulation}

The proponents of the ability model of emotional intelligence have argued that emotional abilities should be measured using ability tests that require respondents to solve problems, rather than using selfreport questionnaires or other measures that mix personality traits, motivation, and skills (Mayer, Salovey, \& Caruso, 2008). Research using ability tests developed by these authors (e.g., the MSCEIT; Mayer, Salovey, \& Caruso, 2002; Mayer, Salovey, Caruso, \& Sitarenios, 2003) has yielded evidence that emotional intelligence is associated with positive outcomes in the realm of social interaction (reviewed by Mayer, Roberts, \& Barsade, 2008). However, most studies reported to date examined a single global score for emotional intelligence, rather than separate scores for emotion regulation and other abilities. Although the four abilities encompassed by Mayer and Salovey's (1997) model can be thought to represent a general factor of emotional intelligence (Mayer et al., 2003), this is not a highly cohesive domain of ability. In fact, correlations between emotion regulation ability and the other three dimensions range from .32 to .51 (Mayer et al., 2002), suggesting that it might be useful to consider emotion regulation as a distinct ability.

One section of the MSCEIT measures strategic emotion regulation ability (SERA) by asking respondents to identify effective strategies for managing emotions in self and others in various situations depicted in brief vignettes. This measure, used in the present studies, is similar to other situational judgment tests in that it reflects judgment or the ability to evaluate situations as well as knowledge (Weekley \& Ployhart, 2006), and different insofar as it focuses on managing emotional situations. It is likely to tap into conscious strategy selection processes more so than automatic processes of emotion regulation operating below conscious awareness (e.g., 
Bargh \& Williams, 2007). Higher scores on this measure were associated with less conflict and antagonism among friends (Lopes et al., 2004, Study 1) and with higher peer ratings of interpersonal sensitivity and prosocial tendencies (Lopes, Salovey, Côté, \& Beers, 2005) — even after controlling for the Big Five personality traits.

\section{Research Questions and Hypotheses}

Distinct forms of emotion regulation may influence different dimensions of social relationship quality. In the present studies, we examined two dimensions of relationship quality - a positive dimension, encompassing warmth, nurturance, companionship, and intimacy; and a negative dimension, encompassing conflict, tension, and antagonism - because these have been found to represent different factors (Furman \& Buhrmester, 1985). We hypothesized that strategic emotion regulation, which draws upon knowledge and cognition, may help people to preempt fruitless conflict or attenuate the negative impact of personal antagonisms more than it helps to enhance the positive dimension of relationship quality, which reflects social bonding and the shared experience of positive emotions. This positive dimension may be influenced more by personality characteristics (such as cheerfulness, extraversion, and emotional expressiveness) and automatic processes of emotion regulation (such as automatic deployment of attention to positive stimuli and automatic appraisal of events in a positive light) that amplify the experience and expression of positive affect and enhance empathy.

Emotion regulation can influence the quality of social interaction in at least two ways. More directly, people can modify the (emotional and social) situation or interaction, attenuating conflict and managing others' emotions directly, independently of one's own affect. More indirectly, people can enhance their own affective experience, which then influences others' affective experience and the emotional tone of the interaction, engendering sociability and positive responses from others through emotional contagion, social appraisal, and interpersonal reinforcement in a positive feedback loop (Parkinson et al., 2005). We reasoned that strategic emotion regulation influences the quality of social relationships by modifying the emotional and social interaction and modulating others' emotions directly, rather than indirectly through the emotional contagion of one's own affect. To evaluate to what extent the latter indirect 
process is important, we examined whether participants' own affect mediated zero-order relationships between SERA and the quality of social relationships. These analyses were intended to further our understanding of strategic emotion regulation and to clarify implications for intervention in the realm of emotional skills training.

To determine whether a construct such as SERA is useful, it is important to ascertain whether it explains variance in criteria over and above well-established constructs. To evaluate the incremental validity of SERA and possible third variable effects, we included the Big Five personality traits in all studies. The Big Five provide a broad map of intrapersonal and interpersonal functioning reflecting emotional reactivity and self-regulation (Larsen, 2000). Extraversion is associated with trait positive affect and social dominance, Neuroticism with trait negative affect, and Agreeableness with low hostility. Controlling for the Big Five therefore represents a stringent test of incremental validity. Because scores on the situational judgment test used in these studies reflect the extent to which respondents agree with experts, in Study 1 we also examined whether the effects of SERA could be explained by socially desirable responding. Considering that a situational judgment test of emotion regulation requires reading comprehension, and although SERA has been found to correlate only weakly with indicators of cognitive ability among college students (e.g., Lopes et al., 2005), in Study 3 we controlled for verbal ability to address concerns that effects might be due to overlap with this indicator of general cognitive aptitude.

There are gender differences in emotional abilities (LaFrance \& Banaji, 1992; Shields, 2002), and some studies suggest that emotional intelligence is related more strongly to social adjustment for men than it is for women (e.g., Brackett, Mayer, \& Warner, 2004). Therefore, we examined whether gender moderates relationships between SERA and the quality of social interaction in the present studies. We also controlled for age (despite restriction of range among our participants) because people are thought to develop emotional skills through learning and experience.

The few studies conducted to date that have found relationships between SERA and the quality of social interaction have relied mostly on North American samples, raising concerns that findings might not generalize to other nations and cultures. Lopes et al. (2004; Study 2) examined such a relationship in a German sample, but the findings did not apply to all types of social interaction. It is 
therefore important to investigate further the link between SERA and social interaction outside North America. The present studies, based on German, Spanish, and American samples, were not designed to test hypotheses about broad cultural differences per se and can provide only a limited test of cross-cultural validity. Nonetheless, at the least, they can enhance our confidence that findings generalize to other Western nations.

In fact, Germany, Spain, and the United States differ in terms of important cultural dimensions such as individualism versus collectivism, power distance, uncertainty avoidance, and masculinity versus femininity (by 1 to 1.8 standard deviations, based on a sample of nations from five continents; Hofstede, 2001). For example, Americans are more individualistic than Germans, who in turn are more individualistic than Spaniards. Germans and Americans also score lower on power distance than Spaniards (see also Hofstede \& McCrae, 2004). Such differences can influence the way people manage their own and others' emotions and react to tense interpersonal situations. For example, being assertive, direct, and upfront when standing up for one's rights may be deemed more acceptable in individualistic than in collectivistic cultures (Morris, Podolny, \& Ariel, 2001).

\section{Overview of the Three Studies}

We examined relationships between SERA, measured with the MSCEIT, and the quality of social interaction or social relationships in three countries: Germany, Spain, and the United States. In the first two studies we collected trait measures of social relationship quality, and in the final study we measured the quality of social interaction and affect through daily reports. In all three studies, we examined whether relationships between SERA and the quality of social interaction were mediated by affect. As indicators of the quality of social interaction, we investigated friendship quality in the first two studies and the quality of everyday social encounters in the third study. Having good friends is related to well-being, adjustment, and satisfaction with life, and also predicts how individuals develop over time (e.g., Hartup \& Stevens, 1997; Helliwell \& Putnam, 2004; Ryff, 1989). Good friendships are associated with instrumental and emotional support, trust, understanding, intimate communication, and closeness. Moreover, friendships are important in both personal and work realms, as having friends at work has been associated with job satisfaction 
(Winstead, Derlega, \& Montgomery, 1995) and can be considered a form of social capital (Helliwell \& Putnam, 2004). We measured both positive and negative dimensions of friendship quality (social support and conflict) because these have been found to represent two distinct factors (Furman \& Buhrmester, 1985). To reduce our reliance on selfreport measures, we collected data from participants and their friends. The third study examined the quality of everyday social encounters involving friends and other people, so as to consider social interaction more broadly and not limit our investigation to friendship.

\section{STUDY 1}

In the first study, we examined the following hypotheses and research questions. First, we expected that SERA would be positively related to indicators of friendship quality, particularly to indicators of conflict and antagonism with friends. Second, we expected that this relationship would remain significant after controlling for age, the Big Five personality traits, and socially desirable responding. Third, we examined whether the relationship between SERA and friendship quality was mediated by trait positive and negative affect.

\section{Method}

\section{Participants}

Participants were 177 first-year German students (115 women and 62 men) enrolled in an introductory course in personality psychology at Chemnitz University of Technology in Germany. Ages ranged from 18 to 41 years $\left(M_{\text {age }}=22.04, S D=3.95\right)$.

\section{Measures}

The present study focused on the following measures, drawn from a data set that included additional scales for separate studies. All measures that were translated into German for this study were also back-translated into English by a different translator so that the quality of the translation could be assessed.

Demographics. Participants reported age and gender.

Strategic emotion regulation ability. SERA was measured with the emotion regulation subscale (Branch 4) of the Mayer-Salovey-Caruso 
Emotional Intelligence Test (MSCEIT V.2.0; Mayer et al., 2002). This measure asks respondents to rate the effectiveness of different strategies for dealing with emotionally challenging intrapersonal and interpersonal situations depicted in eight brief vignettes. It includes 29 items, using a 1 (very ineffective) to 5 (very effective) response format. We used the German version of the test (Schütz, Hertel, \& Schröder, 2002). The full MSCEIT assesses the four abilities composing emotional intelligence as defined by Mayer and Salovey (1997): perceiving emotions in self and others, using emotions to facilitate thought, understanding emotional information, and regulating emotions in self and others.

MSCEIT scores are standardized based on consensus or expert norms ( $M=100, S D=15$, split-half reliability $=.81$ for the normative sample). Expert scores reflect the agreement between a participant's responses and those provided by a sample of 21 experts (i.e., researchers) on emotion. For example, if a participant answers " $A$ " and $21 \%$ of the expert sample also chose that response option, the participant earns a score of .21 for that item. Consensus scores reflect the degree of agreement between a participant's responses and those provided by a normative sample of more than 5,000 people from various nations (mostly North American). The two scoring methods correlate highly (Mayer et al., 2003; $r=.89$ for emotion regulation in the present sample). In this study we used expert norms because they may be less susceptible to cultural bias, and the expert panel was more internationally diverse than the normative sample. In the present sample, the split-half reliability of this scale (corrected by the Spearman-Brown formula) was .65 , and the mean score was 93.8 $(S D=7.73)$.

The vignettes used to measure intrapersonal regulation concern preserving one's own good mood, managing anger following an unfair promotion decision, and managing anxiety about financial difficulties, among other issues. The vignettes used to measure interpersonal regulation concern maintaining a good relationship with a work colleague who is leaving, dealing with a teacher who is not doing enough to help one's son make progress in school, and maintaining good relationships with friends when one feels happy and proud about one's life and achievements. Both sets of vignettes describe emotional states and suggest the challenge involved in managing these. Additional information and sample items appear in Lopes et al. (2005) and Mayer et al. (2003).

Friendship quality. Friendship quality was measured with an abridged version of the Network of Relationships Inventory (NRI; Furman \& Buhrmester, 1985). The full measure consists of 30 items assessing three dimensions: social support, conflict and antagonism, and power imbalance. Given our hypotheses and time constraints, participants answered 
13 items ( 7 for social support and 6 for conflict and antagonism) about their relationship with each of three friends. Respondents used a 9-point Likert-type scale ranging from 1 (not at all) to 9 (extremely). The social support scale, henceforth labeled "supportive friendships" or "positive interaction with friends," included items such as "how much does your friend really care about you?" and "how much do you talk about everything with your friend?" $(M=7.04, S D=0.73, \alpha=.84)$. The scale measuring conflict and antagonism included items such as "how much do you and your friend get upset with or mad at each other?" and "how much do you and your friend argue with each other?" $(M=3.00, S D=1.07$, $\alpha=.90$ ). Each friend completed the same measure about his or her relationship with the participant (for support, $M=6.87, S D=0.70, \alpha=.84$; for conflict, $M=2.90, S D=0.86, \alpha=.88$ ). We also measured the duration and perceived importance of these friendships and contact frequency.

Personality traits. The revised NEO Personality Inventory (NEO-PI-R; Costa \& McCrae, 1992; German version by Ostendorf \& Angleitner, 2004) consists of 240 items and uses a 5-point Likert-type scale anchored at 0 (totally disagree) and 4 (totally agree) to assess the Big Five model of personality: Neuroticism $(M=1.94, S D=0.51, \alpha=.94)$, Extraversion $(M=2.37, S D=0.44, \alpha=.91)$, Openness $(M=2.57, S D=0.37, \alpha=.88)$, Agreeableness $(M=2.34, S D=0.38, \alpha=.89)$, Conscientiousness $(M=$ 2.37, $S D=0.44, \alpha=.93)$.

Trait positive affect was measured with the positive emotions subscale of the NEO-PI-R $(M=2.76, S D=0.68, \alpha=.83)$. Trait negative affect was measured by averaging the 32 items included in the anxiety, depression, angry hostility, and vulnerability to stress subscales of the NEO-PI-R neuroticism factor $(M=1.82, S D=0.59, \alpha=.94)$.

Socially desirable responding. The Balanced Inventory of Desirable Responding (BIDR; Paulhus, 1994; German version by Musch, Brockhaus, \& Bröder, 2002) includes two 10-item subscales (response format: $1=$ strongly disagree to $7=$ strongly agree). Self-deceptive enhancement measures positively biased self-descriptions that participants believe to be accurate (e.g., "my first impressions of people usually turn out to be right"; $M=4.15, S D=0.75, \alpha=.71$ ). Impression management measures people's tendency to misrepresent themselves deliberately to others (e.g., "I never swear"; $M=3.65, S D=0.95, \alpha=.72$ ).

\section{Procedure}

Participants received partial course credit for participating in the study. They took the MSCEIT in a group setting and completed a battery of 
self-report measures at home. They were instructed to ask three good friends (whom they had known for at least 6 months and with whom they did not have a romantic relationship) to rate them on measures of friendship quality. Friends returned the questionnaires in sealed envelopes either by mail or through the participants.

\section{Results}

\section{Preliminary Analyses}

Each participant was asked to obtain ratings from three friends $(N=531 ; 62 \%$ female, $38 \%$ male; mean age 23.4 years, $S D=6.6$ ). Participants reported that they had been friends with these informants for an average of 6.3 years $(S D=4.2)$. They also reported that these friendships were important to them $(M=8.1, S D=1.1$, on a $1-$ 9 scale anchored at $1=$ not important at all and $9=$ very important) and that they met these friends quite often $(M=6.7, S D=1.7$, on a $1-9$ scale anchored at $1=$ very seldom and $9=$ very often). This suggests that the informants knew the participants well.

There was partial agreement between friends' ratings of relationship quality (intraclass correlations were .29 for NRI social support, .27 for NRI conflict, both $p s<.01$ ). Friends' ratings (aggregated across three informants) correlated strongly with participants' self ratings: $r=.51$ for social support and .48 for conflict (both $p s<.01$ ), which further suggests that friends' ratings are meaningful. Preliminary analyses revealed that measures of conflict with friends were significantly skewed. To attenuate skewness, these were transformed by taking the base-10 logarithm.

\section{Strategic Emotion Regulation Ability and Friendship Quality}

Relationships between SERA and self-report indicators of friendship quality were examined with multiple regression analyses that included SERA (standardized), gender (coded female $=0$, male $=1$ ), and the interaction of SERA and gender as independent variables. The top part of Table 1 shows standardized coefficients for the corresponding three effects.

Relationships between SERA and friends' perceptions of friendship quality were examined using a series of multilevel models (HLM, Version 6.06; Raudenbush, Bryk, Cheong, Congdon, \& $\mathrm{du}$ Toit, 2004). These models were the functional equivalent of 


\section{Table 1}

Study 1 (Germany): Relationships Between Emotion Regulation Ability and Indicators of Friendship Quality

\begin{tabular}{lccc}
\hline & Main Effect & Main Effect & Interaction \\
& SERA & Gender & SERA $\times$ Gender \\
\hline Self-Perceptions of Friendship Quality (OLS & Regression) & \\
Positive interaction with friends & .11 & $-.16^{*}$ & -.15 \\
Conflict with friends & $-.25^{* *}$ & .06 & .04 \\
Friends' Perceptions of Friendship & Quality & (Multilevel Analyses) \\
Positive interaction with friends & -.05 & .03 & -.01 \\
Conflict with friends & -.05 & -.16 & $-.19^{*}$ \\
\hline
\end{tabular}

Note. $N=114$ to 115 women, due to missing data, and 62 men. SERA = strategic emotion regulation ability. Gender was coded female $=0$, male $=1$. For ease of interpretation, all variables except gender were standardized prior to analyses. We report standardized coefficients for OLS regression and unstandardized coefficients of fixed effects for multilevel analyses.

$* p<.05$. ** $p<.01$.

conducting a multiple regression with the average of the friends' ratings as a dependent measure. In these analyses, friends' ratings were nested within individuals. At Level 1, the dependent variable (DV) was modeled as a function of an intercept (the average rating for each participant) and a random error term:

$$
\mathrm{y}_{\mathrm{ij}}=\beta_{0 \mathrm{j}}+\mathrm{r}_{\mathrm{ij}}
$$

The Level 1 intercept for each person was then modeled at Level 2 as a function of SERA, gender, the interaction of SERA and gender, and a random error term:

$$
\beta_{0 \mathrm{j}}=\gamma_{00}+\gamma_{01} * \mathrm{SERA}+\gamma_{02} * \text { Gender }+\gamma_{03} *(\mathrm{SERA} * \text { Gender })+\mathrm{u}_{0 \mathrm{j}}
$$

To facilitate the interpretation of results, all variables except gender were standardized prior to multilevel analyses (because these do not yield standardized coefficients). The results of these analyses are summarized in the bottom part of Table 1, which shows the fixed effects representing regression slopes $\gamma_{01}, \gamma_{02}$, and $\gamma_{03}$. Where the interaction between SERA and gender was significant (as for friends' ratings of conflict), we estimated simple slopes for women and men following the recommendations of Aiken and West (1991). Otherwise we focused on the main effect of SERA. 
As can be seen from Table 1, SERA was negatively related to selfreported conflict with friends. The relationship between SERA and friends' ratings of conflict differed for men and women, and the analysis of simple slopes indicated that it was statistically significant for men only $\left(\gamma_{M}=-.23, t=3.46, p<.01\right)$.

\section{Mediation}

To investigate mediation, we followed the four steps proposed by Baron and Kenny (1986) in all three studies. Mediation entails significant relationships between (1) SERA and the DV, (2) SERA and the mediator, and (3) the mediator and the DV. Furthermore, mediation entails (4) a significant reduction in the relationship between SERA and the DV when the mediator is entered as a control variable. We considered that the first condition was met if the main effect of SERA was significant, or if the interaction between SERA and gender as well as the simple slope for men or women were significant. We considered that the second and third conditions were fulfilled if those relationships were significant (for the relevant gender, if the interaction between SERA and gender was significant). The fourth condition was evaluated by adding the mediator to the regression model used for the previous set of analyses (where the relationship between SERA and the DV was moderated by gender) and examining the reduction in the regression coefficient using the Sobel test.

Based on this procedure, the significant relationships between SERA and conflict with friends identified in Study 1 were not mediated by positive or negative trait affect. Let us consider first the relationship between SERA and self-rated conflict with friends. Mediation by negative affect failed in Step 2: SERA was unrelated to trait negative affect $(\beta=-.07, n s$, in regression moderated by gender). Thus, in Step 4, controlling trait negative affect did not reduce the relationship between SERA and self-reported conflict substantially ( $\beta$ dropped from -.25 to $-.24, p s<.01$; Sobel $z=.72, n s$ ). Mediation by positive affect failed in Steps 2 and 3: SERA was only marginally related to trait positive affect $(\beta=.16, p<.10)$, and trait positive affect was unrelated to self-rated conflict with friends. Thus, in Step 4, controlling trait positive affect did not reduce the relationship between SERA and self-reported conflict either (once again, $\beta$ dropped from -.25 to $-.24, p \mathrm{~s}<.01$ ). 
Now consider the relationship found between SERA and friends' ratings of conflict among men. Mediation by trait negative affect failed in Step 3 because this construct was not significantly related to friends' ratings of conflict among men. Thus, in Step 4, controlling trait negative affect did not reduce the relationship between SERA and friends' ratings of conflict among men $\left(\gamma_{01}=-.23\right.$ unchanged, $p<.01)$. With regard to mediation by trait positive affect, Conditions 2 and 3 were satisfied for men. Nonetheless, in Step 4, controlling trait positive affect did not reduce the relationship between SERA and friends' ratings of conflict among men appreciably $\left(\gamma_{01}\right.$ dropped from -.23 to $-.22, p s<.01)$.

\section{Incremental Validity}

We used OLS and multilevel regression analyses to examine whether SERA explained significant variance in indicators of friendship quality, controlling for age, the Big Five, and socially desirable responding (self-deceptive enhancement and impression management). The relationship between SERA and self-rated conflict with friends remained significant $(\beta=-.21, t=2.48, p<.05)$. SERA also remained a significant predictor of friends' ratings of conflict among men $(\gamma=-.16, t=2.35$, $p<.05)$.

\section{Discussion}

SERA was significantly and negatively related to indicators of conflict with friends but not to the positive dimension of friendship quality. This supports our argument that the strategic dimension of emotion regulation may influence conflict and antagonism more than it influences the positive aspects of social relationship quality. SERA was negatively related to self-perceived conflict with friends and to friends' ratings of conflict (among men). These relationships were not mediated by participants' own trait positive and negative affect. Although these findings do not establish causality, they suggest that SERA influences conflict and antagonism by modifying the emotional and social interaction and modulating others' emotions rather than through the contagion of one's own affect.

Relationships between SERA and indicators of friendship quality remained significant after controlling for age, the Big Five personality traits, and socially desirable responding. This suggests that the effect 
of SERA on conflict cannot be explained by overlap with relevant interpersonal characteristics such as Agreeableness or social dominance and assertiveness (aspects of Extraversion). From a practical perspective, it is important to know that SERA explains variance in important aspects of social adaptation over and above established personality constructs. The fact that the effects observed here could not be explained by socially desirable responding attenuates concerns about the use of a situational judgment test that measures the degree of agreement between respondents' judgments of how to regulate emotions and expert judgments.

Although relationships between SERA and indicators of friendship quality were not strong (explaining about $5 \%$ of the variance in outcomes), a review of 125 meta-analyses indicates that small effects should be expected when outcomes are influenced by numerous factors (Meyer et al., 2001). Small effects may also be due to measurement issues, such as the modest reliability of MSCEIT emotion regulation ability found in the present study, restriction of range in ratings of friendship quality (e.g., $54.2 \%$ of self-ratings of positive interaction with friends lay between 7 and 9 on a 1-9 scale), and low inter-rater agreement among friends. Nonetheless, this study yielded evidence of the criterion and incremental validity of a situational judgment test of emotion regulation.

\section{STUDY 2}

Study 2 was designed to replicate and extend the results of Study 1 in Spain. We examined the same hypotheses and research question, obtained a larger sample, and administered some different measures (positive relations with others and trait affect).

\section{Method}

\section{Participants}

Participants were 314 fourth-year psychology students ( 255 women, 59 men) at the University of Málaga. They were White/Caucasian and Spanish, with a mean age of 22.4 years $(S D=3.7)$.

\section{Measures and Procedure}

The data for the present study were drawn from a larger data set that included additional measures for different research purposes. Data were 
collected in two waves, using partially overlapping sets of questionnaires; $53.5 \%$ of participants completed the study in Wave 1 and the remainder in Wave 2. To measure friendship quality and SERA, we used the same measures as in Study 1 in both waves. For the Big Five, different scales were administered in the two waves of data collection, and therefore we standardized scores within wave of data collection. The present study also included self-report measures of trait affect and of the overall quality of social relationships that were not used in Study 1.

All participants completed a battery of tests and questionnaires over three classes of a 4th-year psychology course. Scales that had not been previously validated in Spain were translated into Spanish and then backtranslated into English by an independent translator. Participation was voluntary and anonymous. Participants were also asked to obtain ratings of friendship quality and other measures from three good friends (whom they had known for at least 6 months and with whom they did not have a romantic relationship). Friends returned the questionnaires to the investigators in sealed envelopes, either by mail or through the participants. All participants received partial course credit and were entered into a raffle of 20 -euro vouchers.

Demographics. Participants reported age and gender.

Strategic emotion regulation ability. As in Study 1, we administered the Emotion Regulation subscale of the MSCEIT (Spanish version by Extremera, Fernández-Berrocal, \& Salovey, 2006; $M=96.31, S D=11.54$ ). The authors of the Spanish version reported a split-half reliability of .81 for scores on this branch, based on expert norms and a sample of 946 Spanish individuals. In the present sample, split-half reliability (corrected by the Spearman-Brown formula) was .59 for expert scores.

Quality of social relationships. Participants rated the quality of their relationship with each of three friends (with whom they were not romantically involved) using an 18-item version of the Network of Relationships Inventory (NRI; Furman \& Buhrmester, 1985). These ratings were aggregated across targets, yielding indicators of self-perceived social support $(M=7.42$, $S D=0.89, \alpha=.88)$ and conflict/antagonism $(M=2.73, S D=1.64, \alpha=.94)$. As in Study 1, we also obtained friends' ratings on this measure (social support $M=7.10, S D=0.95, \alpha=.93$; conflict $M=2.49, S D=1.05$, $\alpha=.91)$. In Wave 2 we also measured general satisfaction with social relationships using the Positive Relations with Others subscale of Ryff's (1989) scales of psychological well-being $(M=4.85, S D=0.59, \alpha=.78)$. We administered a 14-item version recommended by the author, with a 6-point response scale anchored at 1 (strongly disagree) and 6 (strongly agree). 
Trait positive and negative affect. Trait positive and negative affect were measured in Wave 2 with the 20-item PANAS (Watson, Clark, \& Tellegen, 1988; Spanish version by Sandín et al., 1999; positive affect $M=3.34, \quad S D=0.67, \alpha=.86$; negative affect $M=2.38, \quad S D=0.78$, $\alpha=.88$ ). Participants indicated to what extent they felt each emotion during the previous year.

Big Five personality traits. Different measures of the Big Five personality traits were administered in the two waves of data collection. Scores were standardized within wave of data collection to yield a common metric, and Cronbach's alphas reported below represent the average of the two measures used. Wave 1 included the Big Five Inventory (BFI-44; John \& Srivastava, 1999; Spanish version by Benet-Martínez \& John, 1998 ; $3.06 \leq M \leq 3.84,0.52 \leq S D \leq 0.79, .66 \leq \alpha \leq .85$ ), a 44-item selfreport inventory using a 5-point Likert-type scale anchored at 1 (disagree strongly) and 5 (agree strongly). Wave 2 included the NEO Five-Factor Inventory (NEO-FFI; Costa \& McCrae, 1992; Spanish version by Cordero, Pamos, \& Seisdedos, 1999; $3.00 \leq M \leq 3.66,0.47 \leq S D \leq 0.70$, $.64 \leq \alpha \leq .87)$, which consists of 60 items using a 5-point Likert-type scale anchored at 0 (totally disagree) and 4 (totally agree).

\section{Results}

\section{Preliminary Analyses}

Although participants were asked to obtain ratings from three friends, 118 were rated by three friends, 75 by two friends, 46 by one friend, and 75 by none. Raters were $62 \%$ female, and their mean age was 22.8 years $(S D=3.6)$. The mean rating for social support was 7.5 (self reported) and 7.1 (as rated by friends), confirming that, in general, informants were good friends. Because participants were rated by a variable number of friends, we estimated inter-rater reliability using the Average Deviation Index (ADI; Burke \& Dunlap, 2002). The ADI represents the mean of absolute deviations among ratings (averaged across raters and across items), calculated in the metric of the original scale. Lower deviations represent higher interrater agreement. The average deviation index was .72 for NRI Positive Interaction and .74 for NRI Conflict, indicating that, on average, raters diverged by about .7 points on a 9-point scale. This is below the cut-off value of 1.5 that Burke and Dunlap recommended for a 9-point scale and therefore indicates high agreement among raters. Note that aggregated friends' ratings correlated significantly 
with self-ratings ( $r=.35$ for positive interaction and .20 for conflict, $p<.01$ ), suggesting that friends' ratings captured meaningful variance in relationship quality.

Measures of social relationship quality were significantly skewed. To attenuate skewness, ratings of conflict were transformed by taking the base-10 logarithm, as in the first study. For Positive Interaction with Friends and Positive Relations with Others, which were negatively skewed, we reflected scores, took the square root, and then reflected scores again so that high scores would indicate positive interaction.

\section{Strategic Emotion Regulation Ability and Friendship Quality}

We followed the analytical strategy used in the previous study. First, we examined relationships between SERA and indicators of friendship quality, moderated by gender, using OLS regression for selfreported DVs and multilevel analyses for friend-rated DVs. The results are summarized in Table 2. SERA was significantly and negatively related to self-perceived conflict with friends but was

\section{Table 2}

Study 2 (Spain): Relationships Between Emotion Regulation Ability and Indicators of Friendship Quality

\begin{tabular}{lccc}
\hline & \multicolumn{2}{c}{ Main Effect } \\
& SERA & Main Effect & Interaction \\
& Gender & SERA × Gender \\
\hline Self-Perceptions of Friendship Quality & (OLS Regression) & \\
Positive interaction with friends & $.16^{*}$ & $-.21^{* *}$ & $-.17^{*}$ \\
Conflict with friends & $-.16^{*}$ & -.09 & .01 \\
Positive relations with others & $.26^{*}$ & -.14 & -.10 \\
Friends' Perceptions of Friendship & Quality (Multilevel Analyses) & \\
Positive interaction with friends & $.13^{*}$ & -.14 & -.17 \\
Conflict with friends & .03 & .20 & -.08 \\
\hline
\end{tabular}

Note. $N=297$ for self-ratings of friendship quality, and 122 for positive relations with others, a measured administered only in one of the two waves of data collection. For friends' ratings of friendship quality, $N=239$. The main effect of SERA is equivalent to the simple slope for women. Gender was coded female $=0$, male $=1$. For ease of interpretation, all variables except gender were standardized prior to multilevel analyses. We report standardized coefficients for OLS regression and unstandardized coefficients of fixed effects for multilevel analyses.

$* p<.05$. ** $p<.01$. 
unrelated to friends' ratings of conflict. SERA was also positively related to self-perceived positive interaction with friends and positive relations with others, as well as to friends' ratings of positive interaction. The relationship between SERA and self-perceived positive interaction with friends was moderated by gender. Examination of simple slopes indicated that it was significant for women but not for men.

\section{Mediation}

We examined whether relationships between SERA and indicators of friendship quality were mediated by trait positive and negative affect, following the steps outlined in Study 1. Note that measures of trait affect were administered only in the second wave of data collection and completed by 146 participants. These analyses indicated that trait affect was not a significant mediator.

In examining the second condition for mediation, which entails a significant relationship between predictor and mediator, regression analyses moderated by gender indicated that SERA was significantly related to trait positive affect $(\beta=.19, p<.05)$ but unrelated to trait negative affect $(\beta=-.07, n s)$. The pattern was similar for correlational analyses combining men and women. This ruled out trait negative affect as a mediator. The third condition for mediation requires that the relationship between mediator and DV be significant. Trait positive affect was significantly related to self-rated positive interaction with friends $(\beta=.22, p<.05)$ but not to friends' ratings of positive interaction $\left(\gamma_{01}=.02, n s\right)$ or to self-rated conflict with friends $(\beta=-.08, n s)$. This ruled out the latter two DVs as candidates for mediation. In the fourth step, controlling trait positive affect reduced the relationship between SERA and self-rated positive interaction with friends (from $\beta=.14$ to .09 , both $n s$ ) and the Sobel test indicated that the mediation effect was marginally significant $(z=1.69, p<.10)$. Note that the relationship between SERA and self-rated positive interaction with friends was weaker (and nonsignificant) in the subsample used for these mediation analyses than the relationship reported in Table 2 based on the full sample. Thus, we found no clear evidence of mediation.

\section{Incremental Validity}

Next, we examined whether relationships between SERA and indicators of friendship quality remained significant after controlling for 
age and the Big Five personality traits. In these analyses, SERA remained significantly and negatively related to self-perceived conflict with friends $(\beta=-.13, p<.05)$; to self-perceived positive interaction with friends, though only for men $\left(\beta_{M}=-.27, p<.05\right)$; and to friends' ratings of positive interaction $\left(\gamma_{01}=.12 ; p<.05\right)$. The relationships between SERA and positive relations with others did not reach statistical significance $(\beta=.14, p=.11)$, possibly due to the lower sample size for this analysis (as this DV was assessed in only one of two waves of data collection).

\section{Discussion}

The results of Study 2 were broadly consistent with those of Study 1 . Among Spanish women, SERA was related to all indicators of the quality of interpersonal relationships except friends' ratings of conflict. These relationships were not mediated by trait positive and negative affect. These relationships remained significant after controlling for age and the Big Five personality traits. Once again, these findings support the notion that SERA influences the quality of social relationships by modifying emotional and social interaction directly, rather than through emotional contagion of a person's own affect. They also suggest that these effects cannot be explained by basic interpersonal characteristics mapped by the Big Five personality traits. Taken together, the results of the first two studies also suggest that relationships between SERA and the quality of social relationships observed in North American samples (e.g., Lopes et al., 2004, 2005) generalize to Western Europeans.

There were some differences between the results of our first and second studies. In particular, in Study 2 we found no evidence that SERA was more strongly related to conflict than to the positive dimension of friendship quality. We will use a meta-analytic synthesis of findings from several studies (below) to test this proposition with adequate statistical power.

Overall, we found more significant relationships between SERA and indicators of social relationship quality in Spain than in Germany. This could be due to cultural differences that could be explored through further research. For example, Spaniards are more emotionally expressive than Germans (Scherer, Wallbott, \& Summerfield, 1986), and it is conceivable that strategic emotion regulation has greater impact in a context of higher emotional 
expressiveness. For self-perceived positive interaction with friends and friends' ratings of conflict, the pattern of results was actually similar across the two studies (as revealed by further analyses of simple slopes for men and women), although some effects did not reach statistical significance in Germany. The relationship between SERA and self-perceived positive interaction with friends appeared to be positive for women but not for men in both studies (although only one of the four simple slopes was significant) - an issue that may also warrant further research.

Our first two studies had some limitations. For example, modest agreement across raters may have limited our ability to detect significant associations with friends' ratings of relationship quality, and we could not control for cognitive ability. The following study addressed these limitations.

\section{STUDY 3}

The present study sought to address the limitations of the first two studies and extend our prior findings. Whereas the previous studies relied on trait-level measures, in this study we examined day-to-day fluctuations in the quality of social encounters and affect using a diary approach. Additionally, we controlled for verbal ability as an indicator of general cognitive ability.

The hypotheses and research questions were similar to those explored in the previous studies: (a) SERA is positively related to the quality of daily social encounters; (b) SERA is positively related to the quality of daily social encounters after controlling for age, gender, the Big Five, and verbal ability; and (c) does daily affect mediate the relationship between SERA and the quality of everyday social interaction?

\section{Method}

\section{Participants}

Undergraduate students at Yale University were recruited from introductory psychology courses and a residential college, and offered course credit or \$20, respectively, for participating in this study. Out of 64 individuals who completed baseline measures, 2 did not complete the second part of the study and 9 did not provide a sufficient number of daily reports (i.e., at least four timely reports) for analysis -7 provided only one report. An invalid report was one that was provided after 10:00 a.m. 
of the following morning or before 9:00 pm of the intended day of a report. This left 53 participants with complete data: 35 women, 18 men. Ages ranged from 18 to $27(M=19.8, S D=1.8)$, with an average of 12.2 days completed $(\mathrm{SD}=2.7)$; $91 \%$ of participants completed 7 or more days. Most participants identified themselves as Caucasian $(72 \% ; 13 \%$ Asian or Asian American, 5\% African American, 9\% “other"). Most were native English speakers (87\%), and the remainder had spent at least 4 years in English-language schools or countries.

\section{Procedure}

Participants completed a battery of tests and questionnaires in the laboratory during the autumn semester, and they participated in the diary study 4 months later. They were asked to log on to a secure Web site and respond to a Web-based questionnaire once a day, before going to sleep, for 14 days. Each daily report took about 10 to 20 minutes to complete. Most participants chose to receive daily email reminders so they would not forget to complete their reports. Participants who missed an entry were notified by email the following day.

\section{Measures of Individual Differences}

Strategic emotion regulation ability. SERA was measured using the corresponding subscale of the MSCEIT (V.2.0; Mayer et al., 2002), scored based on expert norms, as in previous studies $(M=97.0 ; S D=13.1$; splithalf reliability corrected by the Spearman-Brown formula $=.57$ ). Verbal ability was measured using the Mill Hill vocabulary scale for adults, a 66item test of crystallized verbal intelligence (Raven, Court, \& Raven, 1994; $M=44.14 ; S D=5.72$; split-half reliability corrected by the SpearmanBrown formula $=.75)$. The Big Five personality factors were measured using the 44-item Big Five Inventory (BFI-44; John \& Srivastava, 1999; Neuroticism $M=3.15, \mathrm{SD}=0.81, \alpha=.82$; Extraversion $M=3.22, S D=0.91$, $\alpha=.88$; Openness $M=3.74, S D=0.72, \alpha=.84$; Agreeableness $M=3.71$, $S D=0.68, \alpha=.80$; Conscientiousness $M=3.61, S D=0.79, \alpha=.88$ ).

\section{Diary Study Measures}

Participants provided reports of daily events, affect, and the quality of social interactions. For the main outcome variables of interest, we estimated scale reliability using multilevel models that separated variability across items, days, and individuals. Below we report the reliability of the Level 1 coefficient in three-level unconditional models in which items were nested within days, and days were nested within persons (Nezlek, 2007). 
Modest reliabilities, which are typical of diary studies and reflect the use of short measures to prevent participant fatigue and attrition, are offset by an intensive repeated-measures design involving 14 days of data collection.

Daily events. Every day, participants indicated whether 13 events occurred and rated their importance using a 5-point scale $(0=$ did not happen; $1=$ not very important or meaningful; $2=$ somewhat important $/$ meaningful; $3=$ important/meaningful; $4=$ very important/meaningful). This measure yielded two scores measuring the perceived importance of positive social events (seven items; e.g., "had especially good interactions with friend(s) or acquaintances") and negative social events (six items; e.g., "got along poorly with peers - e.g., classmates, co-workers, roommates"). See Nezlek (2005) for a discussion of this scale.

Quality of social interactions. Conflict and tension in social interactions included four items: conflict/tension with close others, conflict/tension with other people, anxiety in social situations, and social exclusion (e.g., "how much tension or conflict did you experience with people you feel close to?"). This 7-point scale was anchored at 1 (not at all) and 7 (very $m u c h$, and its reliability was .45. The quality of interactions with friends, roommates, and acquaintances was measured with three questions (e.g., "how did you get along with friends?") using a 7-point scale anchored at 1 (very badly) and 7 (very well), and its reliability was .65. Participants could also indicate "did not happen/not applicable."

Daily positive and negative affect was measured through participants' responses to the question "how did you feel today?" using a 7-point scale anchored at 1 (did not feel this way at all) and 7 (felt this way very strongly). Positive affect included four items (happy, alert, enthusiastic, relaxed) and its reliability was .43 . Negative affect included five items (stressed, anxious, angry, sad, depressed) and its reliability was .51.

\section{Results}

Altogether, the 53 participants provided 644 reports of daily events $(M=12.2, S D=2.7)$. We used multilevel models to analyze this hierarchically nested data structure, consisting of daily records nested within persons. See Nezlek (2001) for a discussion about analyzing diary study data. To facilitate the interpretation of results, all independent variables (except gender) were standardized prior to the analyses. 


\section{Main Analyses}

We followed the analytical strategy used in the previous studies (although in the present study Level 1 measures represented multiple self-ratings rather than ratings from multiple friends). First, we examined relationships between SERA and mean daily measures, moderated by gender. In these multilevel analyses, an outcome such as perceived conflict was modeled at Level 1 (daily events) as a function of an intercept for each person and a random error term:

$$
\mathrm{y}_{\mathrm{ij}}=\beta_{0 \mathrm{j}}+\mathrm{r}_{\mathrm{ij}}
$$

The Level 1 intercept for each person was then modeled at Level 2 (individual differences) as a function of a constant, SERA, gender, the interaction of SERA and gender, and a random error term:

$$
\beta_{0 \mathrm{j}}=\gamma_{00}+\gamma_{01} * \text { SERA }+\gamma_{01} * \text { Gender }+\gamma_{01} *(\text { SERA } * \text { Gender })+\mathrm{u}_{0 \mathrm{j}}
$$

In Table 3 we report the fixed effects for the slopes indicating the strength of the relationship between predictors and outcomes (e.g., the expected change in a mean daily measure for a $1 \mathrm{SD}$ increase in SERA). These analyses revealed that SERA was negatively related

\section{Table 3}

Study 3 (United States): Relationships Between Emotion Regulation Ability and Daily Measures of Social Interaction

\begin{tabular}{lccc}
\hline & $\begin{array}{c}\text { Main } \\
\text { Effect }\end{array}$ & $\begin{array}{c}\text { Main } \\
\text { Effect } \\
\text { SERA }\end{array}$ & $\begin{array}{c}\text { Interaction } \\
\text { Gender }\end{array}$ \\
SERA $\times$ Gender \\
\hline $\begin{array}{l}\text { Quality of interactions with friends, } \\
\text { roommates, and acquaintances }\end{array}$ & .14 & .00 & -.08 \\
Conflict and tension in social interaction & $-.20 * *$ & .29 & -.05 \\
Importance of positive social events & .02 & .04 & -.13 \\
Importance of negative social events & $-.20 * *$ & .12 & -.06 \\
\hline
\end{tabular}

Note. $N=53$ individuals at Level 2 and 644 daily reports at Level 1 . We report multilevel analyses of fixed effects (the average slope across individuals) and corresponding $t$-tests based on robust standard errors. For ease of interpretation, all variables except gender were standardized prior to multilevel analyses. A separate analysis was conducted for every dependent variable. The main effect of SERA is equivalent to the simple slope for women because gender was coded female $=0$, male $=1$. $p<.05 .{ }^{* *} p<.01$. 
to conflict/tension in social interaction and the importance of negative social encounters, but unrelated to indicators of positive interaction with others. Gender did not moderate relationships between SERA and daily measures of social interaction.

We estimated an effect size for the relationship between SERA and conflict/tension by comparing the between-person variance of this dependent variable in a model with no predictors and in a model including only SERA as a predictor (Nezlek, 2001). SERA explained $19.6 \%$ of the between-person variance in conflict, which is equivalent to a correlation of .44 and represents a medium to strong effect. Note that the dependent measures were not standardized in the present study.

Because our measure of conflict/tension with others included items concerning social anxiety and social exclusion, we checked whether the relationship between SERA and the aggregate of two items that explicitly concerned conflict and tension with others was also negative and significant — and it was.

\section{Mediation and Incremental Validity}

We examined whether daily affect (Level 1) mediated the relationship between SERA and indicators of social interaction. SERA was negatively related to daily negative affect among women but unrelated to positive affect for either gender. Relationships between SERA and both conflict/tension and the perceived importance of negative social events were unchanged $\left(\gamma_{01}=-.21\right.$ and -.20 , respectively) and remained statistically significant $(p s<.01)$ when daily negative affect was added to the models. Thus, there was no evidence of mediation by daily affect.

Next, we examined relationships between SERA and daily measures, controlling for age, the Big Five personality traits, and verbal ability (at Level 2). In these analyses, the relationship between SERA and conflict/tension did not change appreciably but became marginally significant $\left(\gamma_{01}=-.19 ; p=.07\right)$. The relationship between SERA and the perceived importance of negative social events was reduced and no longer significant $\left(\gamma_{01}=-.11, n s\right)$. Further analyses indicated that the reduction in this relationship was primarily due to the inclusion of Agreeableness and Conscientiousness as control variables. 


\section{Discussion}

In a diary study, individuals with high scores on SERA reported less conflict and tension in social interactions and had lower scores for daily negative social events than their counterparts. This replicates and extends the findings from the previous studies, using a research design that is less prone to biases in recall and judgment than ordinary self-report measures. In this study, the relationship between SERA and conflict/tension with others was medium to strong. This attenuates concerns regarding the weaker effect sizes observed in the previous studies and suggests that such weak effects may be partly due to restriction of range on measures of friendship quality.

SERA was unrelated to daily indicators of negative social interaction but not of positive social interaction - a pattern of results similar to that of Study 1. The relationships between SERA and perceived conflict remained marginally significant after controlling for age, gender, the Big Five, and verbal ability. Thus, we found support for the hypothesis regarding criterion validity of SERA but only partial support for incremental validity.

Daily positive and negative affect did not mediate relationships between SERA and conflict or the perceived importance of negative social events. This finding is consistent with those of the previous studies and suggests that strategic emotion regulation influences conflict directly by modifying emotional and social interaction rather than through the emotional contagion of a person's own affect.

There was some attrition in the study, although we do not think it compromises the generalizability of our results. Comparisons of trait-level responses revealed no significant or marginally significant differences between those who provided enough days for analysis and the others, except that the first group scored higher on Neuroticism $(M=3.15$ vs. 2.53). Concerns about attrition are further attenuated by the fact that the present findings are broadly consistent with those of the previous studies.

\section{META-ANALYTIC SYNTHESIS OF FINDINGS}

Across these three studies, SERA was consistently and inversely related to self-perceived conflict and antagonism with friends among women, but less consistently related to the positive dimen- 
sion of friendship, which reflects social support, companionship, nurturance, and intimacy. Among men, relationships between SERA and self-perceived conflict and antagonism with friends were similar to those found for women but did not reach statistical significance, possibly because of the lower number of men in these samples. Thus, we asked ourselves: does SERA help people to manage conflicts more than to enhance companionship and nurturance?

In the first two studies, relationships between SERA and quality of social interaction were not strong, and we lacked the power to determine whether SERA was related more strongly to conflict with friends than to positive interaction with friends. To examine this question more thoroughly, we drew on data described in two previously published articles and one still unpublished data set. These data were from two samples of American students (Lopes et al., 2003, $N=101$; Lopes et al., 2004, Study 1, $N=113$ ) and a Spanish community sample (Cabello \& Fernández-Berrocal, 2008, $N=270$ ). In these studies, participants provided measures of SERA and selfperceived friendship quality (MSCEIT and NRI, the same as in the present Studies 1 and 2). Combined with the data from Studies 1 and 2 from the present article, this allowed us to perform a quantitative synthesis of five studies. These studies involved a total of 963 participants (655 women, 308 men).

Following guidelines for meta-analysis (e.g., Lipsey \& Wilson, 2001), we transformed correlation coefficients using Fisher's $z$ transformation, averaged these across samples weighted by $n-3$, and transformed $z$ scores back into correlations. SERA correlated .12 with positive interaction and -.21 with conflict with friends $(p s<.01)$. The difference between the value of these two dependent correlations, tested using Williams's T2 statistic (as recommended by Steiger, 1980), was statistically significant $(t(960)=2.04, p<.05)$. These findings are consistent with the notion that SERA contributes to both positive and negative dimensions of relationship quality but helps people to manage conflict with friends more than to enhance companionship, nurturance, and intimacy with friends (although we cannot infer causality from the data). We also examined gender differences in relationships between SERA and selfrated friendship quality in the combined data set, and found none. This suggests that the effects observed here apply to both men and women. 


\section{GENERAL DISCUSSION}

We conducted studies in Germany, Spain, and the United States, collected data from self and peers using trait measures and daily reports, and undertook a meta-analytic synthesis of findings that drew upon three additional samples. Considered together, our findings suggest that people who scored higher on a situational judgment test of strategic emotion regulation ability (SERA) tend to experience less conflict with others and have more positive social relationships than their lower-scoring counterparts. Relationships between SERA and various indicators of the quality of social interaction remained significant (or very close to significant) after controlling for age, gender, and the Big Five personality traits (as well as socially desirable responding in one study and verbal ability in another). These results replicate previous findings based on North American samples (e.g., Lopes et al., 2004, 2005) and attenuate concerns about the validity of situational judgment tests of emotion regulation ability, at least within Western cultures. Although we did not examine non-Western cultures, and thus our findings do not constitute a strong test of cross-cultural validity, it should be noted that Germany, Spain, and the United States differ substantially on important and relevant dimensions, including various emotional display rules and norms for social interaction.

Across the three studies, SERA was more consistently related to self-perceived conflict with others than to the positive dimension of social interaction, which reflects social support, companionship, nurturance, and intimacy. A quantitative synthesis that aggregated data from the present studies with additional data from American college students and a Spanish community sample confirmed that emotion regulation ability was more strongly related to conflict with friends than to the positive dimension of friendship quality. Although Studies 1 and 2 found some gender differences in associations between emotion regulation ability and friendship quality (possibly due to sample variation), the quantitative synthesis encompassing additional data revealed no significant gender differences. This suggests that our main findings apply to men and women. Nonetheless, examination of gender differences may warrant further research.

\section{Implications for Theory and Research}

SERA was operationalized as the ability to evaluate emotionally laden situations described in brief vignettes and to identify effective 
strategies for managing emotions in self and others in these situations. This ability also requires judgment integrating emotion and cognition, and the application of intelligence and knowledge to emotional situations. Accordingly, this ability may be more useful for managing conflicts and difficult situations with others than for enhancing companionship, nurturance, and intimacy. To a large extent, the latter aspects of friendship quality reflect liking and positive feelings toward another. They may depend more on natural attraction, rapport, empathy, expressivity, and similarity of interests than on the strategic dimension of emotional intelligence per se (Hartup \& Stevens, 1997). In other words, emotional regulation ability may be more useful for managing conflicts than for enhancing liking among friends.

This interpretation is supported by the fact that in the present studies, positive and negative affect did not mediate the relationship between SERA and conflict, suggesting that SERA does not help people to manage conflicts with others by amplifying positive affect and dampening negative affect in oneself. Instead, the positive interpersonal effects of SERA seem to reflect a more strategic or intellectualized dimension of emotional intelligence, involving the capacity to evaluate emotional situations and identify effective strategies for managing them.

By ruling out an indirect effect based on the emotional contagion of one's own affect, our findings suggest that SERA can influence social relationship quality by other means. These may include modifying the course of an interaction based on one's capacity to evaluate the situation and flexibly select responses appropriate to the situation at hand, abilities that situational judgment tests are designed to measure (see Weekley \& Ployhart, 2006). Although we cannot rule out other possible mediators or third variable effects and cannot infer causality from the data, it is noteworthy that the relationship between emotion regulation ability and conflict could not be explained by the Big Five personality traits, which reflect emotional and interpersonal functioning. For example, Agreeableness is associated with low hostility and Extraversion with gregariousness, social dominance, and emotional expressiveness. Considered together, our findings suggest that the ability to evaluate emotionally challenging interpersonal situations and identify appropriate strategies to manage these situations contributes to the quality of social interaction and helps to explain social adaptation over and above individual differences in basic dimensions of personality. 
Gross (1998b) has argued that emotion regulation is most effective when it takes place before one becomes emotionally aroused. He proposed that regulating emotions through antecedent-focused strategies such as situation modification and cognitive change is more adaptive than controlling behavior or emotional expression in the midst of an intense emotional reaction. We argue that the dimension of emotion regulation ability on which we focused in the present studies is particularly important for antecedent-focused emotion regulation. Specifically, the evaluation of emotional situations and identification of effective response strategies are especially relevant for cognitive appraisal and effective situation modification, which often entail applying intelligence to social and emotional situations. Furthermore, one might expect knowledge of emotion regulation strategies to contribute to reduced conflict in actual relationships through antecedentfocused processes because knowledge alone might not be sufficient to enable down-regulation when someone is in the throes of an emotion.

Whereas most theory and research on emotion regulation focuses on intrapersonal emotion regulation (e.g., Gross, 1998b), our findings highlight the importance of the interpersonal dimension of emotion regulation. Considering that emotions serve communicative and social functions (Keltner \& Haidt, 2001), it may not be possible to disentangle fully emotional and interpersonal regulation in social encounters. According to the theory of emotional intelligence proposed by Mayer and Salovey (1997; Salovey \& Mayer, 1990), emotion regulation ability encompasses skills involved in managing emotions in self and others. In terms of measurement, the emotion regulation branch of the MSCEIT does not contain enough items to separate intrapersonal and interpersonal regulation ability (Mayer et al., 2003). Nonetheless, it may be useful to distinguish intrapersonal and interpersonal emotion regulation in future research (e.g., see Niven, Totterdell, \& Holman, 2009).

Although we measured trait affect in Studies 1 and 2, and daily affect in Study 3, we could not measure the emotions that participants experienced specifically during social encounters. Considering the magnitude of the relationships between SERA and affect experienced within social encounters that was found in a previous study by Lopes et al. (2004, Study 2), it seems plausible that the effects of SERA found in the present studies reflect participants' ability to manage emotional situations in ways that enhance others' emotions and do not depend solely on participants' own affect. 
Further analyses indicated that SERA was only weakly related to trait affect, life satisfaction, and the self-perceived ability to regulate emotion. It is possible that people who are innately predisposed to experience intense negative affect develop emotion regulation skills to compensate for this disposition, as Kagan (1998) suggested. This would dampen relationships between ability measures of emotion regulation and measures of trait affect that reflect the combined effect of temperament and acquired emotion regulation skills. Longitudinal studies are needed to disentangle the effects of temperamental reactivity and emotion regulation skills acquired over time. Studying relationship processes over time may also reveal whether managing conflict effectively can contribute to more positive interactions and social bonding.

The predictive validity of some situational judgment tests is partly due to the fact that scores on these tests also reflect reading comprehension and, more generally, cognitive ability. However, previous research suggests that SERA is unrelated or only weakly related to indicators of cognitive ability among college students, and that relationships between SERA and the quality of social interaction remain significant after controlling for various indicators of cognitive ability (e.g., Lopes et al., 2004, 2005). Similarly, we found that the relationship between SERA and conflict was practically unchanged (although only marginally significant) after controlling for verbal ability in the diary study.

Further research is needed to determine whether the capacity for situational judgment and effective response identification that underlies SERA is domain-specific or generalizes to various realms of life. Preliminary evidence suggests that SERA correlates only weakly with a situation judgment test that was designed to tap into a more global dimension of practical intelligence or common sense (Lopes, 2004), although this result should be interpreted with caution because it could be distorted by the use of different scoring methods for the two tests.

Considered together, our results support the idea that SERA captures important aspects of emotion regulation ability. It is worth noting, however, that situational judgment tests cannot measure all the skills that contribute to effective emotion regulation. For example, a test used in the present studies measures the ability to identify effective strategies for managing emotionally challenging situations rather than people's actual capacity or motivation to regulate 
emotions in life. Further research is needed to determine whether emotion regulation represents a cohesive domain of ability or a weakly interrelated set of skills. It is also important to develop tests of emotion regulation ability that can reliably separate the ability to manage emotions in different types of intrapersonal and interpersonal situations. An additional avenue for further research is to examine interactions between strategic emotion regulation ability and other emotional skills in predicting the quality of social relationships.

In the first two studies, relationships between SERA and indicators of friendship quality were generally small, albeit statistically significant. Yet small effects should not be dismissed when the outcomes are important and determined by multiple factors (as emphasized in a review of 125 meta-analyses by an American Psychological Association task force; Meyer et al., 2001). Having good friends, for example, influences developmental outcomes and well-being throughout life (e.g., Auhagen, 1996; Cacioppo et al., 2008; Hartup $\&$ Stevens, 1997). The quality of social relationships is influenced by emotional and social skills, personality traits, similarity in values and interests, and so on. Therefore we should expect any one factor to explain only limited variance in this outcome. Nevertheless, the relationship between SERA and conflict found in the diary study was moderate to strong. This suggests that intensive repeated measures designs such as the diary method we used may be able to uncover relationships that more traditional methods (e.g., using trait-level measures) cannot.

\section{Limitations}

The modest reliability of MSCEIT emotion regulation ability undermined statistical power to detect significant associations. Nevertheless, lower than optimal reliability also makes hypothesis testing more conservative. The fact that we found a broadly consistent pattern of relationships between emotion regulation ability and indicators of the quality of social relationships and social interaction across three studies despite this limitation increases confidence in our results and also attenuates concerns about Type I error. Although the MSCEIT was not normed in Germany and Spain, the high reliabilities found for the normative sample (Mayer et al., 2003) and a large Spanish sample (Extremera et al., 2006) suggest that the lower 
values found in the present studies were probably due to sampling fluctuations rather than cultural differences.

SERA was negatively related to self-perceived conflict with others in three studies, but less consistently related to friends' perceptions of relationship quality. This could be due to the fact that modest agreement among friends undermined statistical power to detect significant effects on friends' perceptions. Other studies (e.g., Lopes et al., 2004, 2005) also found significant relationships between emotion regulation ability and peer ratings of the quality of social interaction. This suggests that the findings reported here do not just reflect unrealistic self-perceptions or positive illusions. The fact that the relationship between SERA and self-perceived conflict remained significant after controlling for self-deceptive enhancement (in Study 1) also supports this interpretation.

Since the present studies were based on college students, the generalizability of our findings may be limited. Yet research suggests that basic characteristics of good friendships, such as reciprocity and mutuality, are relatively stable through the life span (although characteristics such as what triggers conflict and the types of support provided among friends may change over time; Hartup \& Stevens, 1997). Moreover, it is particularly interesting to study friendship and social interaction among college students because the transition to college often entails making new friends and strains prior relationships (whether or not students continue to live at home, as is often the case in Germany or Spain). The fact that emerging adulthood is marked by the exploration of new interests, identities, and worldviews (Arnett, 2000; Gottlieb, Still, \& Newby-Clark, 2007) can also strain previous friendships, contributing to variability in friendship satisfaction.

\section{Practical Implications}

In light of widespread interest in the enhancement of emotional and interpersonal skills in school and work settings, it is worth highlighting the practical implications of the present studies. Evaluations of school-based programs of social and emotional learning, workbased stress management interventions, and psychotherapy treatments for emotional and interpersonal problems indicate that it is possible to help individuals to manage their emotions and interact with others more effectively (e.g., Aber, Jones, Brown, Chaudry, \& Samples, 1998; Bergin \& Garfield, 1994; Saunders, Driskell, 
Johnston, \& Salas, 1996). Although we cannot infer causality from the present studies, our results are consistent with the notion that training individuals to evaluate emotionally challenging situations and identify effective ways to manage these can enhance their capacity to interact effectively with others and preempt unnecessary conflict. The present results further suggest that this type of training may contribute to the quality of interpersonal relationships in ways that complement training focused on inner emotion regulation. One approach to training that the first author has found useful is to ask participants to discuss in small groups the pros and cons of different strategies for managing emotionally challenging situations, and in what contexts they should opt for one response strategy or another (see also Lopes \& Salovey, 2008). More generally, our findings suggest the importance of combining training that entails applying intelligence to emotionally challenging situations with training aimed at helping people to nurture and express positive emotions in order to enhance bonding and positive relations with others.

\section{REFERENCES}

Aber, J. L., Jones, S. M., Brown, J. L., Chaudry, N., \& Samples, F. (1998). Resolving conflict creatively: Evaluating the developmental effects of a schoolbased violence prevention program in neighborhood and classroom context. Development and Psychopathology, 10, 187-213.

Aiken, L. A., \& West, S. G. (1991). Multiple regression: Testing and interpreting interactions. Newbury Park, CA: Sage.

Arnett, J. J. (2000). Emerging adulthood: A theory of development from the late teens through the twenties. American Psychologist, 55, 469-480.

Arriaga, X. B., \& Rusbult, C. E. (1998). Standing in my partner's shoes: Partner perspective taking and reactions to accommodative dilemmas. Personality and Social Psychology Bulletin, 4, 927-948.

Auhagen, A. E. (1996). Adult friendship. In A. E. Auhagen \& M. V. Salisch (Eds.), The diversity of human relationships (pp. 229-247). New York: Cambridge University Press.

Bargh, J. A., \& Williams, L. E. (2007). The nonconscious regulation of emotion. In J. J. Gross (Ed.), Handbook of emotion regulation (pp. 429-445). New York: Guilford Press.

Baron, R. M., \& Kenny, D. A. (1986). The moderator-mediator variable distinction in social psychological research: Conceptual, strategic, and statistical considerations. Journal of Personality and Social Psychology, 51, 1173-1182.

Benet-Martínez, V., \& John, O. P. (1998). Los Cinco Grandes across cultures and ethnic groups: Multitrait multimethod analyses of the Big Five in Spanish and English. Journal of Personality and Social Psychology, 75, 729-750. 
Bergin, A. E., \& Garfield, S. L. (1994). Handbook of psychotherapy and behavior change (4th ed.). New York: Wiley.

Brackett, M. A., Mayer, J. D., \& Warner, R. M. (2004). Emotional intelligence and its relation to everyday behavior. Personality and Individual Differences, 36, 1387-1402.

Burke, M. J., \& Dunlap, W. P. (2002). Estimating interrater agreement with the average deviation index: A user's guide. Organizational Research Methods, 5, 159-172.

Cabello, R., \& Fernández-Berrocal, P. (2008). Studying emotional intelligence in a Spanish community sample. Unpublished data, University of Málaga.

Cacioppo, J. T., Hawkley, L. C., Kalil, A., Hughes, M. E., Waite, L., \& Thisted, R. A. (2008). Happiness and the invisible threads of social connection: The Chicago Health, Aging, and Social Relations Study. In M. Eid \& R. Larsen (Eds.), The science of well-being (pp. 195-219). New York: Guilford Press.

Caspi, A. (2000). The child is father to the man: Personality continuities from childhood to adulthood. Journal of Personality and Social Psychology, 78, $158-172$.

Cole, P. M., Michel, M. K., \& Teti, L. O. (1994). The development of emotion regulation and dysregulation: A clinical perspective. Monographs of the Society for Research in Child Development, 59, 73-100.

Cordero, A., Pamos, A., \& Seisdedos, N. (1999). Inventario de personalidad NEO revisado (NEO PI-R), Inventario NEO reducido de cinco factores (NEO-FFI) [NEO PI-R Revised Personality Inventory and NEO-FFI Short Five-Factor Inventory]. Madrid: TEA Ediciones.

Costa, P. T., \& McCrae, R. R. (1992). Revised NEO Personality Inventory and NEO Five-Factor Inventory professional manual. Odessa, FL: Psychological Assessment Resources.

Crick, N. R., \& Dodge, K. A. (1994). A review and reformulation of social information-processing mechanisms in children's social adjustment. Psychological Bulletin, 115, 74-101.

Eid, M., \& Diener, E. (2001). Norms for experiencing emotions in different cultures: Inter- and intranational differences. Journal of Personality and Social Psychology, 81, 869-885.

Eisenberg, N., \& Fabes, R. A. (1999). Emotion, emotion-related regulation, and quality of socioemotional functioning. In L. Balter (Ed.), Child psychology: $A$ handbook of contemporary issues (pp. 318-335). Philadelphia: Psychology Press.

Eisenberg, N., Fabes, R. A., Bernzweig, J., Karbon, M., Poulin, R., \& Hanish, L. (1993). The relations of emotionality and regulation to preschoolers' social skills and sociometric status. Child Development, 64, 1418-1438.

Eisenberg, N., Fabes, R. A., Guthrie, I. K., \& Reiser, M. (2000). Dispositional emotionality and regulation: Their role in predicting quality of social functioning. Journal of Personality and Social Psychology, 78, 136-157.

Ekman, P. (2003). Emotions revealed: Recognizing faces and feelings to improve communication and emotional life. New York: Times Books.

Extremera, N., Fernández-Berrocal, P., \& Salovey, P. (2006). Spanish version of the Mayer-Salovey-Caruso Emotional Intelligence Test (MSCEIT) Version 2.0: Reliabilities, age and gender differences. Psicothema, 18, 42-48. 
Frijda, N. H. (1988). The laws of emotion. American Psychologist, 43, 349-358.

Furman, W., \& Buhrmester, D. (1985). Children's perceptions of the personal relationships in their social networks. Developmental Psychology, 21, 1016-1024.

Furr, R. M., \& Funder, D. C. (1998). A multimodal analysis of personal negativity. Journal of Personality and Social Psychology, 74, 1580-1591.

Gottlieb, B. H., Still, E., \& Newby-Clark, I. R. (2007). Types and precipitants of growth and decline in emerging adulthood. Journal of Adolescent Research, 22, $132-155$.

Gross, J. J. (1998a). Antecedent- and response-focused emotion regulation: Divergent consequences for experience, expression, and physiology. Journal of Personality and Social Psychology, 74, 224-237.

Gross, J. J. (1998b). The emerging field of emotion regulation: An integrative review. Review of General Psychology, 2, 271-299.

Harker, L., \& Keltner, D. (2001). Expressions of positive emotion in women's college yearbook pictures and their relationship to personality and life outcomes across adulthood. Journal of Personality and Social Psychology, 80, $112-124$.

Hartup, W. W., \& Stevens, N. (1997). Friendships and adaptation in the life course. Psychological Bulletin, 121, 355-370.

Helliwell, J. F., \& Putnam, R. D. (2004). The social context of well-being. Philosophical Transactions of the Royal Society of London, B, 359, 1435-46.

Hofstede, G. H. (2001). Culture's consequences: Comparing values, behaviours, institutions and organizations across nations (2nd ed.). Thousand Oaks, CA: Sage.

Hofstede, G., \& McCrae, R. R. (2004). Culture and personality revisited: Linking traits and dimensions of culture. Cross-cultural Research, 38, 52-88.

Isen, A. M. (1987). Positive affect, cognitive processes and social behavior. In L. Berkowitz (Ed.), Advances in experimental social psychology (pp. 203-253). New York: Academic Press.

John, O. P., \& Srivastava, S. (1999). The Big Five trait taxonomy: History, measurement, and theoretical perspectives. In L. A. Pervin \& O. P. John (Eds.), Handbook of personality: Theory and research (2nd ed., pp. 102-138). New York: Guilford Press.

Kagan, J. (1998). Galen's prophecy. Boulder, CO: Westview Press.

Keltner, D., \& Haidt, J. (2001). Social functions of emotions. In T. J. Mayne \& G. A. Bonanno (Eds.), Emotions: Current issues and future directions (pp. 192213). New York: Guilford Press.

LaFrance, M., \& Banaji, M. R. (1992). Toward a reconsideration of the genderemotion relationship. In M. S. Clark (Ed.), Emotion and social behavior: Review of personality and social psychology (Vol. 14, pp. 178-201). Newbury Park, CA: Sage.

Larsen, R. J. (2000). Toward a science of mood regulation. Psychological Inquiry, 11, 129-141.

Lerner, J. S., \& Tiedens, L. Z. (2006). Portrait of the angry decision-maker: How appraisal tendencies shape anger's influence on cognition. Journal of Behavioral Decision Making, 19, 115-137.

Lipsey, M. W., \& Wilson, D. B. (Eds.). (2001). Practical meta-analysis. Thousand Oaks, CA: Sage. 
Lopes, P. N. (2004). Emotional and practical intelligence in college. Unpublished data, Yale University.

Lopes, P. N., Brackett, M. A., Nezlek, J. B., Schütz, A., Sellin, I., \& Salovey, P. (2004). Emotional intelligence and social interaction. Personality and Social Psychology Bulletin, 30, 1018-1034.

Lopes, P. N., Salovey, P., Côté, S., \& Beers, M. (2005). Emotion regulation ability and the quality of social interaction. Emotion, 5, 113-118.

Lopes, P. N., \& Salovey, P. (2008). Emotional intelligence and leadership: Implications for leader development. In C. L. Hoyt, G. R. Goethals, \& D. R. Forsyth (Eds.), Leadership at the crossroads: Psychology and leadership (Vol. 1, pp. 78-98). Westport, CT: Praeger.

Lopes, P. N., Salovey, P., \& Straus, R. (2003). Emotional intelligence, personality, and the perceived quality of social relationships. Personality and Individual Differences, 35, 641-658.

Lyubomirsky, S., King, L., \& Diener, E. (2005). The benefits of frequent positive affect: Does happiness lead to success? Psychological Bulletin, 131, 803-855.

Mayer, J. D., Roberts, R. D., \& Barsade, S. G. (2008). Human abilities: Emotional intelligence. Annual Review of Psychology, 59, 507-536.

Mayer, J. D., \& Salovey, P. (1997). What is emotional intelligence? In P. Salovey \& D. J. Sluyter (Eds.), Emotional development and emotional intelligence: Educational implications (pp. 3-31). New York: Basic Books.

Mayer, J. D., Salovey, P., \& Caruso, D. R. (2002). Mayer-Salovey-Caruso Emotional Intelligence Test (MSCEIT) user's manual. Toronto, ON: MHS.

Mayer, J. D., Salovey, P., \& Caruso, D. (2008). Emotional intelligence: New ability or eclectic traits? American Psychologist, 63, 503-517.

Mayer, J. D., Salovey, P., Caruso, D. R., \& Sitarenios, G. (2003). Measuring emotional intelligence with the MSCEIT V2.0. Emotion, 3, 97-105.

Meyer, G. J., Finn, S. E., Eyde, L. D., Kay, G. G., Moreland, L. K., \& Dies, R. R. (2001). Psychological testing and psychological assessment: A review of evidence and issues. American Psychologist, 56, 128-165.

Mischel, W., \& DeSmet, A. L. (2000). Self-regulation in the service of conflict resolution. In M. Deutsch \& P. T. Coleman (Eds.), The handbook of conflict resolution: Theory and practice (pp. 256-275). San Francisco: Jossey-Bass.

Morris, M., Podolny, J., \& Ariel, S. (2001). Culture, norms, and obligations: Cross-national differences in patterns of interpersonal norms and felt obligations toward coworkers. In W. Wosinska, R. Cialdini, D. Barrett, \& J. Reykowski (Eds.), The practice of social influence in multiple cultures (pp. 97-123). Mahwah, NJ: Erlbaum.

Musch, J., Brockhaus, R., \& Bröder, A. (2002). Ein Inventar zur Erfassung von zwei Faktoren sozialer Erwünschtheit [An inventory for the assessment of two factors of social desirability]. Diagnostica, 48, 121-129.

Nezlek, J. B. (2001). Multilevel random coefficient analyses of event and interval contingent data in social and personality psychology research. Personality and Social Psychology Bulletin, 27, 771-785.

Nezlek, J. B. (2005). Distinguishing affective and non-affective reactions to daily events. Journal of Personality, 73, 1539-1568. 
Nezlek, J. B. (2007). Multilevel modeling in research on personality. In R. Robins, R. C. Fraley, \& R. Krueger (Eds.), Handbook of research methods in personality psychology (pp. 502-523). New York: Guilford Press.

Niven, K., Totterdell, P., \& Holman, D. (2009). A classification of controlled interpersonal affect regulation strategies. Emotion, 9, 498-509.

Ostendorf, F., \& Angleitner, A. (2004). NEO-Persönlichkeitsinventar nach Costa und McCrae, Revidierte Fassung (NEO-PI-R) [NEO Personality Inventory by Costa and McCrae, revised version]. Göttingen: Hogrefe.

Parkinson, B., Fischer, A. H., \& Manstead, A. S. R. (2005). Emotion in social relations: Cultural, group, and interpersonal processes. Hove, UK: Psychology Press.

Paulhus, D. L. (1994). Balanced Inventory of Desirable Responding: Reference manual for BIDR version 6. Vancouver, Canada: University of British Columbia.

Raudenbush, S., Bryk, A., Cheong, Y. F., Congdon, R., \& du Toit, M. (2004). HLM 6: Hierarchical linear and nonlinear modeling. Lincolnwood, IL: Scientific Software International.

Raven, J. C., Court, J. H., \& Raven, J. (1994). Manual for Raven's progressive matrices and vocabulary scales. Oxford: J. C. Raven.

Ryff, C. D. (1989). Happiness is everything, or is it? Explorations on the meaning of psychological well-being. Journal of Personality and Social Psychology, 57, 1069-1081.

Salovey, P., \& Mayer, J. D. (1990). Emotional intelligence. Imagination, Cognition, and Personality, 9, 185-211.

Sandín, B., Chorot, P., Lostao, L., Joiner, T. E., Jr., Santed, M., \& Valiente, R. (1999). Escalas PANAS de afecto positivo y negativo: Validación factorial y convergencia transcultural [PANAS positive and negative affect scales: Factorial validity and cross-cultural convergence]. Psicothema, 11, 37-51.

Saunders, T., Driskell, J. E., Johnston, J. H., \& Salas, E. (1996). The effect of stress inoculation training on anxiety and performance. Journal of Occupational Health Psychology, 1, 170-186.

Scherer, K. R., Wallbott, H. G., \& Summerfield, A. B. (1986). Experiencing emotion: A cross-cultural study. New York: Cambridge University Press.

Schütz, A., Hertel, J., \& Schröder, M. (2002). MSCEIT Version 2.0 Deutsche Bearbeitung. Chemnitz, Germany: Chemnitz University of Technology.

Shields, S. A. (2002). Speaking from the heart: Gender and the social meaning of emotion. Cambridge: Cambridge University Press.

Simon, H. A. (1967). Motivational and emotional controls of cognition. Psychological Review, 74, 29-39.

Steiger, J. H. (1980). Tests for comparing elements of a correlation matrix. Psychological Bulletin, 87, 245-251.

Thompson, R. A. (1994). Emotion regulation: A theme in search of definition. Monographs of the Society for Research in Child Development, 59, $25-52$.

Watson, D., Clark, L. A., \& Tellegen, A. (1988). Development and validation of brief measures of positive and negative affect: The PANAS scales. Journal of Personality and Social Psychology, 54, 1063-1070. 
Weekley, J. A., \& Ployhart, R. E. (Eds.). (2006). Situational judgment tests: Theory, measurement, and application. Mahwah, $\mathrm{NJ}$ : Erlbaum.

Winstead, B. A., Derlega, V. J., \& Montgomery, M. J. (1995). The quality of friendships at work and job satisfaction. Journal of Social and Personal Relationships, 12, 199-215.

Zillmann, D. (1993). Mental control of angry aggression. In D. M. Wegner \& J. W. Pennebaker (Eds.), Handbook of mental control (pp. 370-392). Englewood Cliffs, NJ: Prentice-Hall. 